\title{
AN AUTOMATED SKIN DISEASE DIAGNOSTIC SYSTEM BASED ON DEEP LEARNING MODEL
}

\section{LAWRENCE OMOTOSHO ${ }^{1}$, KEHINDE SOTONWA ${ }^{2}$, BENJAMIN ADEGOKE ${ }^{3}$, OLUWASHINA OYENIRAN ${ }^{4}$, JOSHUA OYENIYI $^{1 *}$}

\author{
${ }^{1}$ Department of Information and Communication Technology, Osun State University, \\ Osogbo, Osun State, Nigeria \\ ${ }^{2}$ Department of Computer Science and Information Technology, Bells University of \\ Technology, Ota, Ogun State, Nigeria \\ ${ }^{3}$ Department of Computer Engineering, Federal Polytechnic, Ile-Oluji, Ondo \\ State, Nigeria \\ ${ }^{4}$ Department of Computer Science, Ajayi Crowther University, Oyo, Oyo State, \\ Nigeria
}

\begin{abstract}
The use of computer technology has significantly advanced the medical sector, and many computer technologies have been used to develop healthcare, such as the patient management system, monitoring and control systems, and diagnostic systems. Technological advances in healthcare have also helped in saving numerous patients and are constantly improving our quality of life. Technology in the medical sector has also had a major effect on almost all healthcare professional techniques and practices. In order to facilitate rapid diagnosis and treatment of different skin diseases by the use of a deep learning model, this study developed a comprehensive framework to improve the decision-making of dermatologists in Nigeria in terms of the diagnosis of selected skin diseases. The developed system achieved the network accuracy of $98.44 \%$ and the validation accuracy of the test set is $99.44 \%$ as specified by the training results, further testing reveal that the developed system yielded rejection rate of $2.2 \%$ and recognition accuracy of $97.8 \%$.
\end{abstract}

Keywords: dermatology, artificial intelligence, deep learning, AlexNet

\section{INTRODUCTION}

Artificial intelligence (AI) has recently experienced a notable exponential development in many sectors, and health care is no exception [1]. AI is especially useful in healthcare and will fundamentally change the pathways of diagnosis and treatment for many ailment and diseases [2]. The general purpose of the application of this technology in medicine, irrespective of the particular methodology, is to use computer algorithms to discover relevant data knowledge and to support clinical decision-making [3]. The application of AI technology in healthcare has developed rapidly in many developed countries and it is considered to have increases human resources and skills thereby improving the accuracy of medical treatment. As many countries supporting the development of advanced technologies welcome the application of AI, the necessary governance specifications by

\footnotetext{
${ }^{*}$ Corresponding author, email: thejoshoyeniyi@ gmail.com

(C) 2021 Alma Mater Publishing House
} 
law, regulation, technology and standards will begin to be developed in order to fully optimize this developing field of technology [2]. Thus, this study seeks to see the relevance of AI in the field of dermatology.

Dermatology is the medical specialty that deals with diseases and conditions affecting the skin, hair, scalp. However, a larger part of dermatology focuses on skin issues and treatments. This is because the skin, which is the body's most extensive organ, is more prone to a wider range of diseases and conditions [4]. People of all ages, from newborns to elderly people, are all prone to skin problems that come in several different forms, from uncommon to normal and from chronic to brief problems [5].

There are various of skin diseases with four types of skin conditions as Chronic diseases, Acute skin problems, Skin infections, Skin discolorations. Skin conditions that arise briefly and unexpectedly but cause serious symptoms relate to acute skin problems [4]. Cold sores, Warts, Hair loss, Blisters, Corns and calluses, Sunburn, Ingrown hair, Dermatitis, Itching, Hives, Lice, Bruises and Sebaceous cysts are the most common acute skin issues. Skin infections are reaction to infectious irritants, fungi, and bacteria. Carbuncles, boils, cysts, abscesses, leprosy, staph infection, and cellulitis are common bacterial infections that affect the skin. Ringworm, athlete's foot, yeast infection, fungal nail infection, and sporotrichosis are fungal diseases of the skin [5]. On the other hand, common viral infections include shingles, chicken pox and Molluscum Contagiosum [4].

Skin discolorations such as birthmarks, moles, freckles and skin tags are also dealt with by dermatologists. There are color-varying patches or stains on the skin. In certain ways, discoloration is innate. They can also evolve later in life, however. Birthmarks, moles, and freckles do not need medical care most of the time [5]. However, in some situations, a dermatologist can interfere either because of their height, positioning, or other symptoms felt by the patient. Genetics and exposure to the sun are common sources of skin discoloration. Cryosurgery or freezing and removing, electrosurgery or burning and removing, and laser treatments are widely used treatments.

Skin disease has become a popular disease among humans in recent times [6]. These diseases typically have concealed risks, contributing not only to a loss of self-confidence and psychological distress, but also to a risk of skin cancer[7]. One of the most infectious diseases to be seen in people is skin illnesses. Because of the physical structure influenced by direct exposure to ultraviolet radiation, i.e. the long-term use of various types of highfrequency wireless equipment can result to development of skin cancer [7]. Because of the symptoms of skin disease, the diagnosis of skin diseases is very difficult due to an extended and constantly transforming phase occurring in some areas of the skin [8]. Many clues, such as basic lesion anatomy, physical assortment, scaling, shadow, and arrangement, may be used to diagnose skin disease. By independently evaluating particular components [6], the recognition process can be very complicated. In general, medical experts with high-level instruments are necessary to diagnose these types of diseases due to the lack of visual resolution in skin disease images [8].

In addition, manual diagnosis of skin disease is often subjective, time-consuming, and more human effort is required [9]. Thus, a computer-aided system that can automatically diagnose skin diseases needs to be created. One of the most infectious diseases to be seen in people is skin illnesses. Nigeria is a developing tropical country located in the western part of sub-Saharan Africa, with about hundred specialist dermatologists servicing a country population of 177 million people, the ratio of a dermatologist to patients is 1:1,770,000 [10]. Atopic dermatitis, tinea, acne, contact dermatitis, urticaria, seborrheic dermatitis, pityriasis versicolor, vitiligo and human papilloma virus infections were also highlighted as the nine most common skin conditions in Nigeria [10]. In this regard, a robust system needs to be developed to not replace dermatologists in Nigeria, but to support their decision-making in the diagnosis of selected skin diseases, so as to promote rapid diagnosis and treatment of different skin diseases. Thus, this study seeks to develop a deep learning model in diagnosis of the nine most common skin disorders in Nigeria as specified by [10].

\section{EMPERICAL REVIEW OF LITERATURE}

In order to carry out this study, it is highly important to critically review all available and relevant literatures in medical image processing so as to establish and validate the pragmatic status of this research work. Thus, this study looks into the related research works, which is the relevant contribution that has been proposed and implemented in medical image processing in relation to dermatology. 
The task of obtaining, evaluating and applying the vast amount of information required to solve complex clinical problems is faced by modern medicine. The advancement of medical artificial intelligence has been connected to the creation of AI programs to help the clinician formulate a diagnosis, make therapeutic choices, and predict outcomes. They are designed to assist healthcare staff in their everyday jobs, helping with tasks that rely on data and information manipulation. Artificial neural networks (ANNs), fuzzy expert systems, evolutionary computation, and hybrid intelligent systems are included in these systems [11]. Neural networks have advanced at a remarkable rate, and they have found practical applications in various fields of study [12]. Deep learning can solve problems which are hardly solvable with traditional artificial intelligence, as it can utilize unlabeled information during training; it is thus well-suited to addressing heterogeneous information and data, in order to learn and acquire knowledge [13].

A large number of newer studies have highlighted the capabilities of advanced deep learning technologies, including learning from complex data, image recognition, text categorization among host of others [13]. One of the main applications of deep learning is for medical diagnosis [14]. This includes but is not limited to health informatics, biomedicine, and magnetic resonance image MRI analysis. Segmentation, diagnosis, classification, prediction, and identification of multiple anatomical regions of interest are more basic applications of deep learning in the medical field. Deep learning is far superior compared to conventional machine learning, as it can learn from raw data and has multiple hidden layers that allow it to learn input-based abstractions [15].

The process for creating and assessing the classification model for dermatological classification was designed [16] with deep learning technique. They employ CNN architecture of AlexNet with transfer learning scenario. This study uses the dermatological images from a public data source. These data are the dermatoscopic images of seven skin lesions related to the disease. In order to broadly investigating the transfer-learning concept, they created three types of models (Alexnet-TL, FESVM, and FESVM+PD, respectively). The experimental results performed on a public dataset show that the CNN model can classify skin diseases with $79.29 \%$ accuracy.

In [17] the effectiveness and capability of convolutional neural networks in the classification of 8 skin diseases was studied. The diagnostic types included in the used dataset includes melanoma, melanocytic nevi, basal cell carcinoma, benign keratosis, actinic and intraepithelial carcinoma, dermatofibroma, vascular lesions, and atypical nevi. The main goal is to compare the potential of deep learning with the performance of dermatologists who are highly educated. The experimental result shows that all models of deep learning model performed better than the dermatologists (at least $11 \%$ ). In total, various pre-trained state-of-the-art architectures such as DenseNet 201, ResNet 152, Inception v3, InceptionResNet v2 were used and applied to 10135 dermoscopy skin pictures (HAM10000: 10015, PH2: 120). 94.40 \% (ResNet 152) and 99.30\% (DenseNet 201) are the highest ROC AUC values for melanoma and basal cell carcinoma, compared with $82.26 \%$ and $88.82 \%$ for dermatologists. DenseNet 201 also had the highest AUC values for the general classification of macro and micro averages $(98.16 \%, 98.79$ $\%$ ) respectively).

A high-precision deep learning neural network for automated diagnosis of skin tumors was created [18]. During the works, a training sample was formed, comprising real photographs of skin lesions and the images obtained from the source through various distortions. The unique augmentation algorithms, coupled with the loss functions, are the main authoring, allowing to achieve a high quality classification of skin diseases with a limited training set. The developed model allows quality diagnosis of melanoma with an accuracy of not less than $91 \%$, which is comparable with the diagnostic capabilities of highly qualified dermatologists. The use of intelligent systems of this type to identify skin diseases will provide substantial support in the diagnosis of both dermatologists and general practitioners.

The accuracy of deep learning algorithms to diagnose three common dermatopathology diagnoses was determined [17]. The developed system accurately classified 123/124 (99.45 \%) BCCs (nodular), 113/114 (99.4 \%) dermal nevi, and 123/123 (100\%) seborrheic keratoses. The authors Concluded that Artificial intelligence using deep learning algorithms is a potential adjunct to diagnosis and may result in improved workflow efficiencies for dermatopathologists and laboratories.

A pre-trained was employed in [19], ResNet-34, ResNet-50, ResNet-101, and ResNet-152 were chosen as the candidate architectures since they exhibit feature re-use and propagation, which were essential to our fine tuning. They normalized data with the recommended mean and standard deviation. The data was split into training and validation sets in in 5:1 ratio after which dynamic in-memory augmentation such as crop, random zoom, horizontal $\&$ vertical flips in the data loader was performed. This showed that from user-submitted images with deep learning 
based classifiers, many skin diseases can be detected. They noted that the accuracy levels become architectural agnostic, provided adequate training and substantial difference exists between error-free detection and peak efficiency reached by modern techniques. Also, the gap cannot be easily bridged since it appears from the nature of the presentation of skin disease. Experimental result showed that the output dipped by at least $10 \%$ in non-ideal conditions such as noise, blur and distribution shift, which are rational scenarios in any field trial and the method presented improve the quality of dermatological services.

A system to classify skin diseases of 10 different classes containing 5500 images obtained from the Dermnet dataset was proposed [20]. The framework proposed consists of 2 parts-image processing and transfer learning for dermatological image processing. The part of image processing deals with the augmentation of images and the removal of unwanted components, which is found to be necessary before further processing, otherwise the performance quality would be affected. And transfer learning component deals with features extractions and fine tuning of pre-trained VGG16 model. The validation accuracy is $74.1 \%$ and by further fine tuning it yielded $76.3 \%$ when tested on those datasets.

In [21] a method that uses techniques related to computer vision to distinguish different kinds of dermatological skin abnormalities was projected. For training and testing purposes, they used different types of Deep Learning algorithms (Inception v3, MobileNet, Resnet, xception) for feature extraction and learning algorithms (preferably Random Forest or Logistic Regression). The use of state-of-the-art architecture dramatically enhances productivity by up to $88 \%$. Furthermore, by using mapping of ensemble functions, combing the models trained using Inception V3, MobileNet, Resnet, Xception would be a voting-based model and thus improve efficiency by using ensemble features mapping13. Logistic regression was employed to optimize efficiency and pick the optimal architecture for the application. The divide mode is set to $90 \%$ for training in this method, $10 \%$ for data validation/testing. A confusion matrix table is used to describe the efficiency of a classification model (or 'classifier') on a collection of test data for which the true values are used.

It is evident that majority of all the aforementioned authors in this empirical study focused on skin cancer classification. The empirical review of this study established that skin cancer is a common concept that interest researchers in diverse field of study which make it not restricted to dermatology alone but also exposed it to computer science and engineering. Thus, this empirical study revealed that numerous researchers in this regard ventured into skin cancer, moreover, various studies have established that larger percentage of skin diseases can lead or cause skin cancer if not well managed.

Hence, this study is tailored to research in to some obvious skin diseases (those that are common to Nigerians), truly, they may not be life threatening but these specified skin diseases have higher tendency of afflicting higher degree of distress to the carriers of such skin diseases. As a result, this study aims to employ pretrained deep learning algorithm in to the classification of these selected diseases, so as to aid effective diagnosis which will foster appropriate treatment of these diseases.

\section{EXPERIMENTAL SETUP}

In order to accomplish the purpose of this study, the development process follows the designed framework as specified in Figure 1. The datasets for the skin diseases classification were built, the images were acquired over the internet by keywords searches. A total of one thousand eight hundred images were obtained for the nine classes which were basically used for training and another two hundred and seventy images were obtained for the nine classes with were used for further testing and evaluation of the model. For the purpose of Image Pre-processing, the acquired images were subjected to basic image preprocessing tasks which include cropping and resizing. In this regards, images in this study were resized to 227 by 277 by 3, which is accepted image size for the AlexNet model.

This study uses the AlexNet pretrained model, where the training dataset (one thousand eight hundred images) were split into $80 \%$ by $20 \%$, where the $80 \%$ is used for training and the remaining $20 \%$ is used for validation upon which the validation accuracy of the developed system is based. Before the training of the model, the pretrained AlexNet was fine-tuned so as to accommodate the specified nine classes. After the training of the model, the new trained model was saved and renamed as DERMNET. The testing datasets (two hundred and seventy images) were used to further test and evaluate the developed system, the result of which was regarded as the overall recognition accuracy of the system. 


\section{RESULTS AND DISCUSSION}

This study presents the application of deep learning model in diagnosis of the nine most common skin disorders in Nigeria, the development is based on the system architecture adopted for this study (see Figure 1) and the basic system requirements (Machine name: DESKTOP-Q2NPMI9, Machine Id: \{5C1CDF56-5C07-4E2F-BB5B6EA9441721FF\}, Operating System: Windows 10 Pro 64-bit (10.0, Build 18363) (18362.19h1_release.1903181202), System Manufacturer: TOSHIBA, System Model: Satellite L650, BIOS: InsydeH2O Version 1.90 (type: BIOS), Processor: Intel(R) Core(TM) i5 CPU M 430 @ 2.27GHz (4 CPUs), 2.3GHz, Memory: 4096MB RAM).

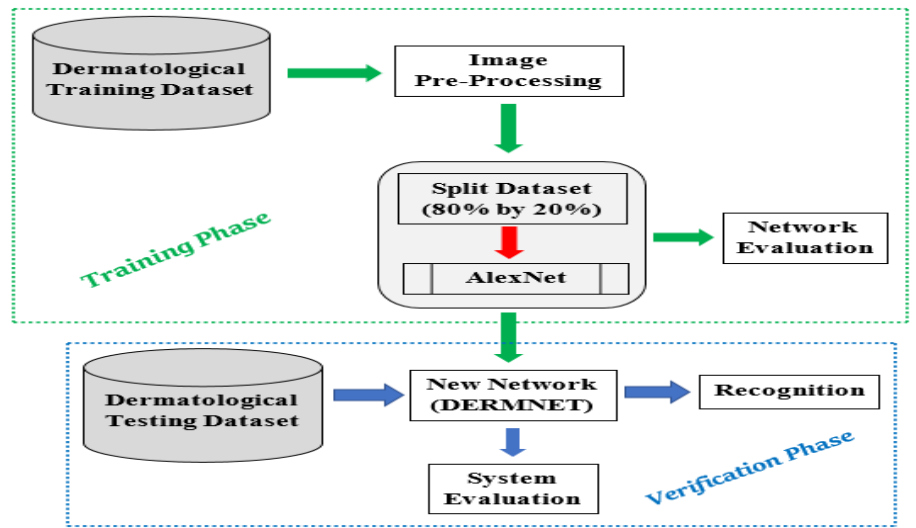

Fig. 1. System Architecture for diagnosing of the nine most common skin disorders in Nigeria.

Figure 2 show the interface of the MATLAB software used for simulation, training and testing. The developed system achieved the network accuracy of $98.44 \%$ as show in Table 1 and the validation accuracy of the test set is $99.44 \%$ as show see Figure 3.

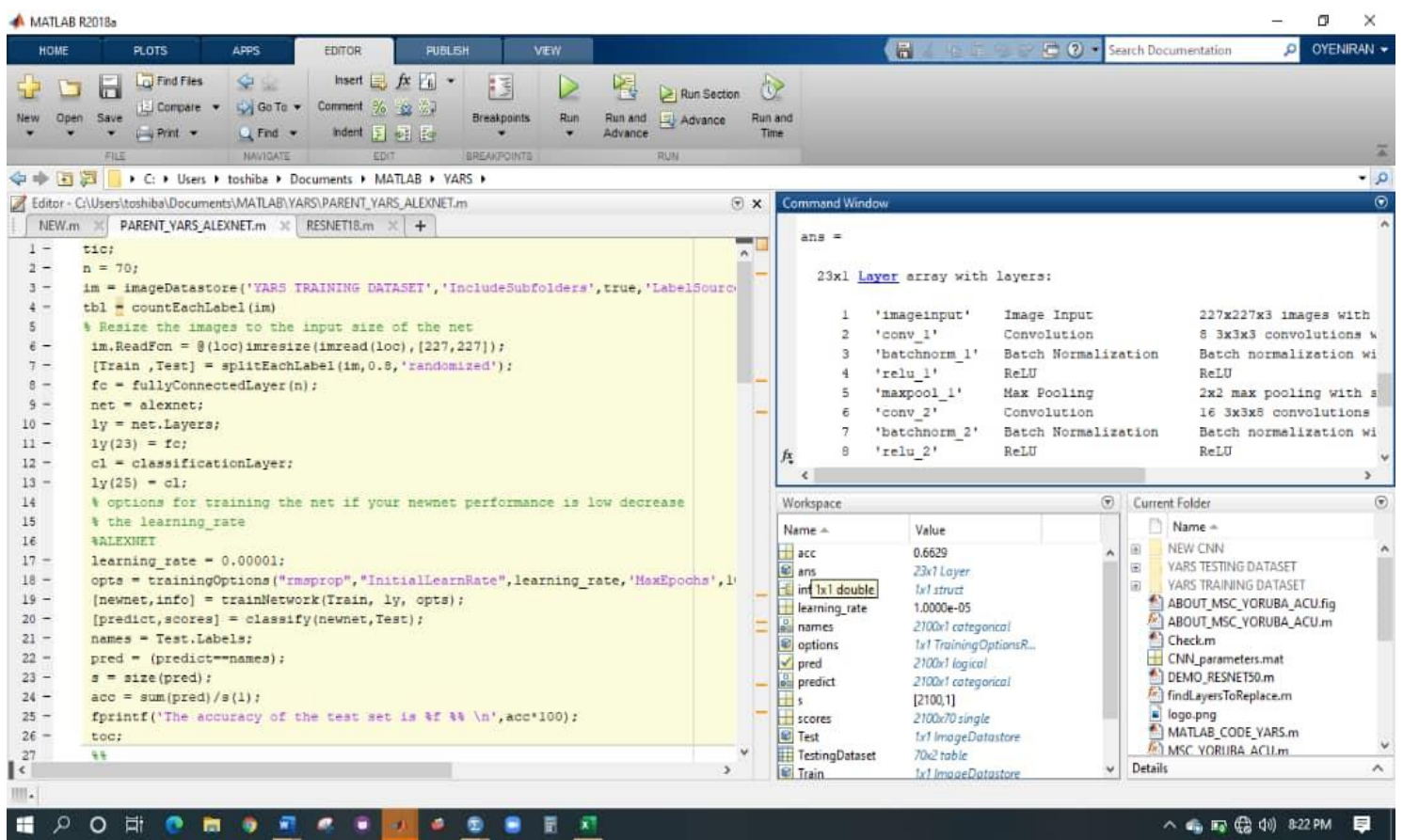

Fig. 2. System development interface.

Furthermore, the model was subjected to further testing using testing dataset (270 images) which yielded rejection rate of $2.2 \%$ and recognition accuracy of $97.8 \%$ as show see Table 2 . Also, The findings of this study with respect to Table 2 indicate that total testing sample for this study is 270 images where each class have 30 images. All the classes yielded $100 \%$ recognition accuracy except for Pityraisis Versicolour, Seborrheic Dermatitis and Tinea with $93.3 \%, 90 \%$ and $96.7 \%$ respectively. Statistical evaluation of the result using descriptive statistics indicates 
that all classes yielded the standard deviation of 0.00000 except for Pityraisis Versicolour, Seborrheic Dermatitis and Tinea with $0.25371 \%, 0.30513$ and 0.18257 respectively (Table 3 ).

Table 1. Training progress.

\begin{tabular}{|l|l|l|l|l|l|}
\hline Epoch & Iteration & $\begin{array}{c}\text { Time Elapsed } \\
\text { (hh:mm:ss) }\end{array}$ & $\begin{array}{c}\text { Mini-batch } \\
\text { Accuracy }\end{array}$ & $\begin{array}{c}\text { Mini-batch } \\
\text { Loss }\end{array}$ & Base Learning Rate \\
\hline 1 & 1 & $00: 00: 09$ & $18.75 \%$ & $2.7835 \%$ & $1.0000 \mathrm{e}-05$ \\
\hline 3 & 50 & $00: 07: 16$ & $71.88 \%$ & $0.9400 \%$ & $1.0000 \mathrm{e}-05$ \\
\hline 5 & 100 & $00: 14: 31$ & $92.19 \%$ & $0.4635 \%$ & $1.0000 \mathrm{e}-05$ \\
\hline 7 & 150 & $00: 21: 46$ & $93.75 \%$ & $0.2022 \%$ & $1.0000 \mathrm{e}-05$ \\
\hline 10 & 200 & $00: 28: 53$ & $100.00 \%$ & $0.0540 \%$ & $1.0000 \mathrm{e}-05$ \\
\hline 10 & 220 & $00: 38: 15$ & $98.44 \%$ & $0.1030 \%$ & $1.0000 \mathrm{e}-05$ \\
\hline
\end{tabular}

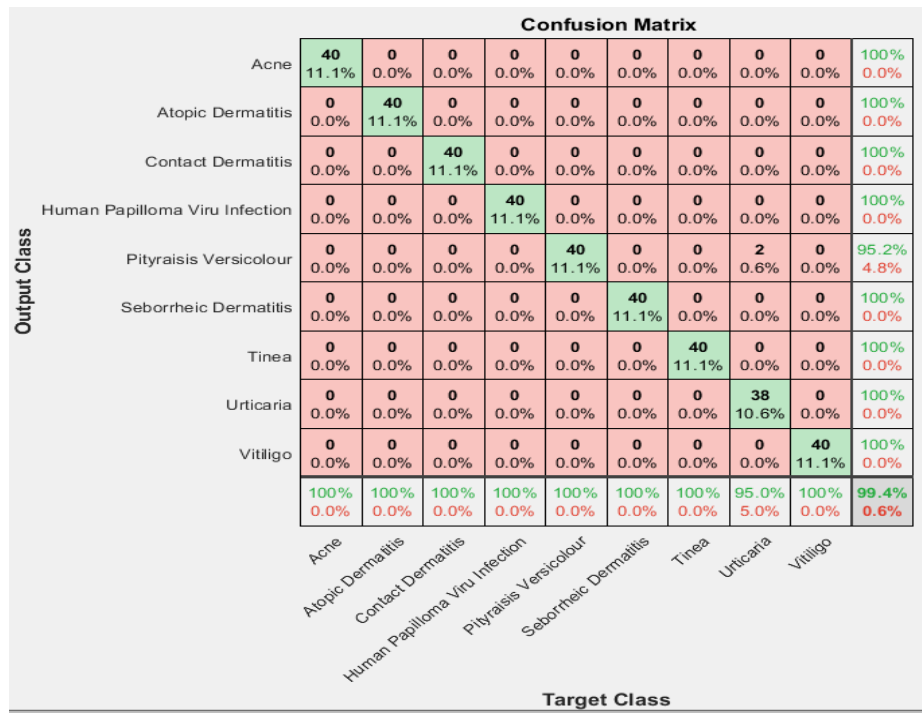

Fig. 3. Confusion Matrix for classification of the nine most common skin disorders in Nigeria.

Table 2. System verification.

\begin{tabular}{|l|c|c|c|c|c|}
\hline Skin Diseases & $\mathrm{N}$ & Minimum & Maximum & Mean & Std. Deviation \\
\hline Acne & 30 & 1.00 & 1.00 & 1.0000 & .00000 \\
\hline Atopic Dermatitis & 30 & 1.00 & 1.00 & 1.0000 & .00000 \\
\hline Contact Dermatitis & 30 & 1.00 & 1.00 & 1.0000 & .00000 \\
\hline Human Papilloma Virus Infection & 30 & 1.00 & 1.00 & 1.0000 & .00000 \\
\hline Pityraisis Versicolour & 30 & 1.00 & 2.00 & 1.0667 & .25371 \\
\hline Seborrheic Dermatitis & 30 & 1.00 & 2.00 & 1.1000 & .30513 \\
\hline Tinea & 30 & 1.00 & 2.00 & 1.0333 & .18257 \\
\hline Urticaria & 30 & 1.00 & 1.00 & 1.0000 & .00000 \\
\hline Vitiligo & 30 & 1.00 & 1.00 & 1.0000 & .00000 \\
\hline
\end{tabular}

Table 3. Descriptive statistics.

\begin{tabular}{|l|c|c|c|c|c|}
\hline \multicolumn{1}{|c|}{ Skin diseases } & $\begin{array}{c}\text { Testing } \\
\text { samples }\end{array}$ & $\begin{array}{c}\text { Correctly } \\
\text { recognised }\end{array}$ & $\begin{array}{c}\text { Incorrectly } \\
\text { recognized }\end{array}$ & Not recognised & Recognition rate \\
\hline Acne & 30 & 30 & 0 & 0 & $100 \%$ \\
\hline Atopic Dermatitis & 30 & 30 & 0 & 0 & $100 \%$ \\
\hline Contact Dermatitis & 30 & 30 & 0 & 0 & $100 \%$ \\
\hline $\begin{array}{l}\text { Human Papilloma } \\
\text { Virus Infection }\end{array}$ & 30 & 30 & 0 & 0 & $100 \%$ \\
\hline Pityraisis Versicolour & 30 & 28 & 02 & 0 & $93.3 \%$ \\
\hline $\begin{array}{l}\text { Seborrheic } \\
\text { Dermatitis }\end{array}$ & 30 & 27 & 03 & 0 & $90 \%$ \\
\hline
\end{tabular}




\begin{tabular}{|l|c|c|c|c|c|}
\hline Tinea & 30 & 29 & 01 & 0 & $96.7 \%$ \\
\hline Urticaria & 30 & 30 & 0 & 0 & $100 \%$ \\
\hline Vitiligo & 30 & 30 & 0 & 0 & $100 \%$ \\
\hline TOTAL & $\mathbf{2 7 0}$ & $\mathbf{2 6 4}$ & $\mathbf{0 6}$ & $\mathbf{0}$ & $\mathbf{9 7 . 8} \%$ \\
\hline
\end{tabular}

\section{CONCLUSION}

This study investigates the application of deep learning in diagnosis of the nine most common skin disorders in Nigeria, with the aim of using AlexNet pretrained deep learning model. The findings of this study indicates that the overall recognition accuracy of the developed system is $97.8 \%$ thus, accomplishing its purpose. Moreover, comparing the results of this study with its contemporaries indicate that this model will aid the diagnosis skills of the Nigerian dermatologists. Since the network is already trained with quality dataset and yielded good network and recognition accuracy. Hence, there is no need for the end user to retrain the network. In order to use the developed system, the user is expected to input an image then subject it to the trained network for diagnosis.

Nevertheless, the recognition accuracy of Pityraisis Versicolour, Seborrheic Dermatitis and Tinea cannot be overlooked, thus, this study recommends that quality and more datasets should be used in future trainings so as to yield better skin diagnosis.

\section{REFERENCES}

[1] He, J., Baxter, S.L., Xu, J., Zhou, X., Zhang, K., The practical implementation of artificial intelligence technologies in medicine, Nature Medicine, no. 25, 2009, p. 30-36.

[2] Xu, J., Xue, K., Zhang, K., Current status and future trends of clinical diagnoses via image-based deep learning, Theranostics, vol. 9, no. 25, 2019, p. 7556-7565.

[3] Murdoch, T., Detsky, A., The inevitable application of big data to health care, Journal of the American Medical Association, vol. 309, 2013, p. 1351-2.

[4] https://www.docdoc.com.sg/info/speci alty/d ermatologists/ (04.08.2020).

[5] Chambers, H., Pharmacology and the treatment of complicated skin and skin-structure infections, The New England Journal of Medicine, vol. 370, 2014, p. 2238-2239.

[6] Siegel, R.L., Miller, K.D, Jemal, A., Cancer statistics, A Cancer Journal for Clinicians, vol. 65, no. 5, 2005 , p. 29.

[7] Robinson, J., Sun exposure, sun protection and vitamin D, The Journal of the American Medical Association, vol. 294, 2005, p.1541-1543.

[8] Belal, A., Mohd, U., Chuen-Min, H., Kai, H., Hossain, M.S., Ghulam, M., Discriminative feature learning for skin disease classification using deep convolutional neural network, International Conference on Environment and Electrical Engineering, IEEE Explore, 2017.

[9] Fabbrocini, G., Vita, V., Cacciapuoti, S., Leo, G., Liguori, C., Paolillo, A., Pietrosanto, A., Sommella, P., Automatic diagnosis of melanoma based on the 7-point checklist, Computer Vision Techniques for the Diagnosis of Skin Cancer, Series in Bio Engineering, 2014, p. 71-107.

[10] Henshaw, E., Ibekwe, P., Adeyemi, A., Ameh, S., Ogedegbe, E., Archibong, J., Olasode, O., Dermatologic practice: Review of common skin diseases in Nigeria, International Journal of Health Sciences and Research, vol. 8, no.1, 2018, p. 235-248.

[11] Ramesh, A.N., Kambhampati, C., Monson, J.R., Drew, P.J., Artificial intelligence in medicine, Annals of the Royal College of Surgeons of England, vol. 86, no. 5, 2004, p. 334-338.

[12] Szegedy, C., Wei, L., Yangqing, J., Sermanet, P., Reed, S., Anguelov, D., Erhan, D., Vanhoucke,V., Rabinovich, A., Going deeper with convolutions, IEEE, Conference on Computer Vision and Pattern Recognition (CVPR), 2005, p. 1-9.

[13] Mihalj, B., Dragica, R., Deep learning and medical diagnosis: A review of literature, Multimodal Technologies and Interaction, vol. 2, no. 3, 2018, no 47.

[14] Lee, J.G., Jun, S., Cho, Y.W., Lee, H., Kim, Seo, J.B., Kim, N., Deep learning in medical imaging: General overview, Korean Journal of Radiology, vol.1, no. 4, 2017, p. 570-584.

[15] Miotto, R., Wang, F., Wang, S., Jiang, X., Dudley, J., Deep learning for healthcare: review, opportunities and challenges, Briefings in Bioinformatics, vol. 19, no. 6, 2018, p.1236-1246. 
[16] Kittipat, S., Supaporn, B., Kittisak, K., Nittaya, K., Dermatological classification using deep learning of skin image and patient background knowledge, International Journal of Machine Learning and Computing, 2019, p. 862-867.

[17] Amirreza, R., Habib, S., Somayeh, K., Dermatologist level dermoscopy skin cancer classification using different deep learning convolutional neural networks algorithms, vol. 317, 2018, p. 257-1484.

[18] Olsen, T.G., Jackson, B.H., Feeser, T.A., Kent, M.N., Moad, J.C., Krishnamurthy, S., Lunsford, D.D., Soans R.E., Diagnostic performance of deep learning algorithms applied to three common diagnoses in dermatopathology, Journal Pathology Informatics, vol. 9, no.11, 2018, p. 32.

[19] Gavrilov, D., Shchelkunov, N., Melerzanov, A., Deep learning based skin lesions diagnosis, ISPRS International Archives of the Photogrammetry, Remote Sensing and Spatial Information Sciences, vol. 52, no. 8, 2019 , p. 81.

[20] Mishra, S., Imaizumi, H., Yamasaki, T., Interpreting fine-grained dermatological classification by deep learning, Proceedings of the IEEE/CVF Conference on Computer Vision and Pattern Recognition (CVPR) Workshops, 2019.

[21] Anil, S., Srijana, B., Sunidhi, A., Madhusudan, M., Subarna, S., Dermatological diseases classification using image processing and deep neural network, International Conference on Computing, Communication, and Intelligent Systems (ICCCIS) 2019, p. 381-386.

[22] Patnaik, S.K., Sidhu, M.S., Gehlot, Y., Sharma, B., Muthu, P., Automated skin disease identification using deep learning algorithm, Biomedical and Pharmacology, vol. 11, no. 3, 2018, p. 1429-1436. 